\title{
Spectinomycin Sulfate Tetrahydrate
}

National Cancer Institute

\section{Source}

National Cancer Institute. Spectinomycin Sulfate Tetrahydrate. NCI Thesaurus. Code C90692.

The tetrahydrate sulfate salt form of spectinomycin, an aminocyclitol aminoglycoside antibiotic derived from Streptomyces spectabilis with bacteriostatic activity.

Spectinomycin binds to the bacterial 30 S ribosomal subunit. As a result, this agent interferes with the initiation of protein synthesis and with proper protein elongation. This eventually leads to bacterial cell death. 